\title{
Practices for the disbudding and dehorning of dairy calves by veterinarians and dairy producers in Ontario, Canada
}

\author{
Charlotte B. Winder, ${ }^{*}$ Stephen J. LeBlanc, ${ }^{*}$ Derek B. Haley, ${ }^{*}$ Kerry D. Lissemore, ${ }^{*}$ M. Ann Godkin, $\dagger$ \\ and Todd F. Duffield*1 \\ *Department of Population Medicine, University of Guelph, Ontario, Canada N1G 2W1 \\ †Ontario Ministry of Agriculture, Food, and Rural Affairs, Elora, Ontario, Canada NOB 1S0
}

\begin{abstract}
Disbudding and dehorning dairy calves is very common, despite the introduction of polled genetics to most dairy breeds. Appropriate pain-control practices for these procedures affect both calf welfare and public perception of the dairy industry. Previously published work has shown that North American dairy producers have not widely adopted use of these medications for disbudding or dehorning. However, since the last published work examining these practices in Canada, changes regarding awareness, availability, and future requirements for pain control have occurred in the industry. With this in mind, online and telephone surveys of both veterinarians $(\mathrm{n}=238)$ and dairy producers $(\mathrm{n}$ $=603$ in Ontario, Canada, were conducted in the fall of 2014 with a goal of describing current disbudding and dehorning practices and examining factors associated with the adoption of pain control use. Approximately three-quarters of dairy producers reported performing disbudding or dehorning themselves, whereas the remainder used a veterinarian or technician. Almost all $(97 \%)$ of the veterinarians surveyed reported using local anesthetic, $62 \%$ used sedation, and $48 \%$ used a nonsteroidal anti-inflammatory drug. Producer use of local anesthetic was $62 \%, 38 \%$ used sedation and $24 \%$ used a nonsteroidal anti-inflammatory drug. Seventyeight percent of veterinarian disbudding or dehorning was done before 8 wk of age, whereas $64 \%$ of dairy producers performed this procedure before $8 \mathrm{wk}$ of age. Seventy-two percent of veterinarians and $63 \%$ of producers reported changing their disbudding or dehorning practices over the past $10 \mathrm{yr}$; of producers that changed their practices, $73 \%$ cited their herd veterinarian as influential. The use of pain control described in these surveys is higher than previously reported in Ontario. Identification of factors associated with best practices,
\end{abstract}

Received April 4, 2016.

Accepted August 3, 2016.

${ }^{1}$ Corresponding author: tduffiel@uoguelph.ca or the lack of adoption of these practices, may help veterinarians target appropriate educational opportunities for their dairy clients.

Key words: disbudding, dehorning, calves

\section{INTRODUCTION}

Horned dairy cattle pose risk of injury to humans and other cattle (Stock et al., 2013). Dehorning or disbudding dairy calves is very common; $94 \%$ of US dairy farms report having dehorned animals (USDA, 2009). Canadian surveys of disbudding and dehorning practices do not report any producer respondents leaving horns intact (Misch et al., 2007; Vasseur et al., 2010), and we suspect the vast majority of calves born on Canadian dairy farms are either disbudded or dehorned. However, these procedures are well documented to cause significant pain when done without appropriate analgesia (Stafford and Mellor, 2011; Stock et al., 2013). Industry stakeholders have identified painful procedures as key welfare issues for dairy cattle (Ventura et al., 2015). Beyond the individual animal, these procedures have an effect at the industry level, as consumers often emphasize freedom from pain as important (Spooner et al., 2014), and the vast majority of stakeholders believe pain relief should be provided for the disbudding and dehorning of dairy calves (Robbins et al., 2015).

Dehorning refers to the removal of the horn after attachment of the horn bud to the skull, occurring at approximately 2 mo of age (CVMA, 2010; AVMA, 2014). Disbudding refers to the removal or destruction of the horn buds before this time.

Disbudding and dehorning are generally done by 1 of 3 methods: surgical amputation of the horn after attachment to the base of the skull, cautery of the horn bud, or the application of caustic paste to the horn bud (Stock et al., 2013). These methods cause different types of damage to the tissue, and may result in different experiences of pain type, intensity, and duration (Petrie et al., 1996; Stilwell et al., 2009; Braz et al., 2012). All of these procedures are painful when done 
without appropriate anesthesia and analgesia (Stafford and Mellor, 2011).

Local anesthesia, commonly given as a cornual nerve block, prevents acute pain, but, when given alone, a protracted rise in physiological and behavioral indicators occurs after the duration of the block (Sylvester et al., 1998; Grøndahl-Nielsen et al., 1999). Addition of a nonsteroidal anti-inflammatory drug (NSAID) reduces pain behaviors (Duffield et al., 2010), extending up to $44 \mathrm{~h}$ after disbudding when injectable meloxicam is used (Heinrich et al., 2010). The use of sedation (e.g., xylazine, an $\alpha$-adrenergic agonist) has been shown to reduce handling stress (Grøndahl-Nielsen et al., 1999) and may improve safety when dehorning older animals or when handling facilities are lacking.

In Canada, the majority of dairy calf disbudding and dehorning is performed by dairy producers or on-farm staff, whereas some farms choose to employ their veterinarian or veterinary technician for these procedures (Misch et al., 2007; Vasseur et al., 2010). Reported pain control use by producers for dehorning in North America ranges from 15 to $40 \%$, although this generally reflects only use of local anesthetic, as reported NSAID use has been very low, if reported at all (Hoe and Ruegg, 2006; Fulwider et al., 2008; Vasseur et al., 2010). Although use of local anesthetic for disbudding and dehorning by veterinarians is quite high (Huxley and Whay, 2006; Misch et al., 2007; Fajt et al., 2011), reported NSAID adoption rates by veterinarians have been much lower (Huxley and Whay, 2006; Misch et al., 2007; Fajt et al., 2011).

Surveys of veterinarians' disbudding and dehorning practices have been done in Ontario in 2004 (Misch et al., 2007), Canada in 2005 (Hewson et al., 2007), as well as the United Kingdom in 2004 (Huxley and Whay, 2006) and the United States in 2007 (Fajt et al., 2011). North American dairy producers' practices have been surveyed in regions of the United States (Hoe and Ruegg, 2006; Fulwider et al., 2008) and Canada (Misch et al., 2007; Vasseur et al., 2010); practices by European dairy producers have also been explored (Gottardo et al., 2011; Staněk et al., 2014; Hokkanen et al., 2015). As these studies use different regions of dairy production, it is not easily discerned if practices have changed over time in any given population. Since the last published work in Canada, a substantial shift has occurred in the industry's awareness of appropriate practices for disbudding and dehorning. The Code of Practice for the Care and Handling of Dairy Cattle (National Farm Animal Care Council, 2009), a voluntary industry code distributed to all dairy producers, requires the use of pain control for these procedures. In the same year, Canada saw licensing and marketing of the first NSAID with a label indication for use following disbudding in dairy calves less than $3 \mathrm{mo}$ of age (Metacam $20 \mathrm{mg} /$ $\mathrm{mL}$ Solution for Injection; Boehringer-Ingelheim, Burlington, Ontario, Canada). Dairy producers are also increasingly aware of a new, mandatory Canadian dairy program (proAction), the animal care portion of which begins rollout in 2017 and will require the use of pain control for dehorning (Dairy Farmers of Canada, 2015).

With these industry changes in mind, our primary objective was to describe current disbudding and dehorning practices, including pain control use, by veterinarians and dairy producers in Ontario. The study also examined self-reported changes over time and sought to explore reasons for, attitudes toward, and factors associated with the adoption of best practices.

\section{MATERIALS AND METHODS}

Two similar surveys, one for Ontario-based bovine veterinarians and one for licensed Ontario dairy producers, were launched in October 2014 and closed in February 2015 after pretesting with 5 veterinarians and 5 dairy producers. Surveys were hosted online (Qualtrics, Provo, UT) with an option to participate via telephone. Online surveys were compatible for access via computer, tablet, or smartphone. Survey content and recruitment methods were approved by the University of Guelph's Research Ethics Board for the use of human subjects in research.

\section{Survey Populations and Survey Delivery}

Veterinarians were recruited from the membership of the Ontario Association of Bovine Practitioners ( $\mathrm{n}=$ 238) through an email distributed through a listserv and postcards distributed at an Ontario Association of Bovine Practitioners meeting in November 2014. Based on the small total number, the entire population was surveyed. We anticipated dehorning practices to be specific to the veterinarian as opposed to the clinic. Based on this assumption, we wanted to reflect total practices occurring in the province and not over-represent the effect of smaller clinics with few veterinarians or underrepresent large clinics with numerous bovine-only veterinarians.

From 3,915 licensed dairy producers with the Dairy Farmers of Ontario (DFO) in 2014, a subset $(\mathrm{n}=603)$ were contacted to participated in the survey. This subset reflected $15 \%$ of each of the 15 geographic regions for the DFO, weighted by herd population within the region, and rounded up by one producer per region. Dairy producers were invited to participate by 2 mailouts, occurring in October 2014 and January 2015. All invitations to participate contained the survey's URL as well as a quick response code (QR code) linking with 
the survey's website for use with a tablet or smartphone, and a phone number to call if the participant wished to complete the survey over the phone.

\section{Survey Design}

Survey questions on disbudding and dehorning practices and pain control use were similar to those asked in a 2004 Ontario survey (Misch et al., 2007), which included sections on individual, farm, and clinic demographics. Both surveys are available to view in the Supplemental Material (http://dx.doi.org/10.3168/ jds.2016-11270). Producers were classified as using a particular disbudding or dehorning method if used for $>50 \%$ of their calves. Three percent of producers reported a 50:50 split between 2 methods and were then defined as being a user of the more invasive method, as a larger diameter burner was considered more invasive than a small diameter burner, and surgical amputation was considered more invasive than burning (Petrie et al., 1996). Producers were asked a dichotomous outcome question on use of pain-control medication for the majority of their disbudding or dehorning, as we expected practices to be fairly uniform on individual farms. In both surveys, participants were given a list of reasons they could select as to why they chose to use or not use local anesthetics, sedatives, or NSAID for disbudding or dehorning. They were able to select multiple reasons, all reasons, or none. An "other" option with an opportunity to write a response was given.

Also included were questions to collect data on potential explanatory variables for disbudding and dehorning practices and use of pain control. Questions on calf management and the use of polled genetics were included in the producer survey. Questions on knowledge of the Code of Practice for the Care and Handling of Dairy Cattle (National Farm Animal Care Council, 2009) and perceptions of pain in cattle were included in both surveys. Perception of pain was evaluated using a 10-point Likert scoring system similar to previous work (Huxley and Whay, 2006; Hokkanen et al., 2015). Both veterinarians and producers were given 8 conditions or procedures applying to cattle, and asked to rate pain based on a 0 to 10 point ordinal Likert scale. Zero was defined as not causing pain, and 10 defined as the worst imaginable pain. It was stated that no pain control was given unless specified. Wording of the procedures and conditions was slightly different based on the 2 audiences. Conditions were dystocia (hard pull with a calving jack), left-displaced abomasum (LDA) surgical correction (with local anesthetic given), severe clinical mastitis (febrile, abnormal milk, sick cow), lameness (acute sole ulcer), ear tagging (newborn calf), disbudding by caustic paste at $2 \mathrm{~d}$ of age, disbudding by propane- or butane-powered small burner at 2 wk of age, and dehorning at 5 mo of age using gouging then using cautery to control bleeding.

\section{Statistical Analysis}

Telephone responses were entered online on behalf of 18 producers, representing $11 \%$ of that survey's respondents. Online data were exported into Microsoft Excel (Microsoft Corp., Redmond, WA) and checked for completeness and errors. Of all survey responses from both groups, none were excluded due to missing data, which was defined as respondents that began the first survey page but did not view the last survey page. Therefore, all responses were included in the final analysis.

For the veterinary survey, data at the clinic-level were obtained by taking the mean response of all respondents who reported working for a single clinic. Clinic of employment was determined through direct reporting and cross-referencing with 2014 licensure information provided by the College of Veterinarians of Ontario (Guelph, Ontario, Canada). Clinic data included information on involvement of registered veterinary technicians in disbudding or dehorning, as well as approximate number of clients utilizing veterinarians or technicians for disbudding or dehorning.

Descriptive statistics were reviewed in STATA13 (Stata/IC Version 13.1 for Mac, StataCorp, College Station, TX). Differences in median Likert scores between the 8 procedures or conditions were assessed separately for each population (veterinarians and producers) using Mood's median test (K-sample equality-of-medians test) for nonparametric data. Differences in equality of distribution between the 2 populations (veterinarians and producers) were assessed for each of the 8 procedures or conditions using the Kolmogorov-Smirnov test (measuring, among other things, differences in range, mean, and variance). For all tests, $P<0.05$ was used as the critical value.

Predictors of medication use among producers were examined by logistic regression modeling. Nonsteroidal anti-inflammatory drug use was not considered for a predictive model, as it was less commonly used and data for analysis would have been too sparse. Therefore, only models for producer use of local anesthetic and sedation were built. Veterinarian data lacked variation for local anesthetic use and, due to the structure of the data, reliable, stable models could not be constructed for veterinarian sedation and NSAID use.

Forty-eight explanatory variables contained $>10 \%$ variation and were organized into 9 categories: individual demographics, farm demographics, veterinary involvement, resource use, technology use, calf management, Likert scoring, disbudding practices, and knowl- 
edge of the Code of Practice for the Care and Handling of Dairy Cattle. Univariate logistic regression with either local anesthetic or sedative use as the dependent variable was performed for all variables. Variables with a $P$-value of $<0.20$ were considered for the multivariate model. If one or more variables per survey section were potentially significant $(P<0.20)$, the variable with the lowest $P$-value was considered for the multivariate models. All variables considered for the multivariate models were checked for collinearity using the Spearman rank coefficient. A pair of variables was considered collinear if the correlation was $>0.70$. Final multivariable models were built by forward manual stepwise selection, with variables kept if their $P$-value was $<0.05$, although herd health frequency was kept in the final sedation model $(P=0.071)$ as it was a significant variable in the local anesthetic model and was considered as a variable of interest. Rejected variables were assessed as confounder effects and kept in the model if their removal changed the coefficients of the remaining variables by more than $20 \%$. All terms in the final model were tested for interactions, but none were found in either of the models. Models were assessed by Hosmer-Lemeshow goodnessof-fit test. The predictive ability of the models was assessed by generating a receiver operator characteristic (ROC) curve and by examining sensitivity and specificity at a probability threshold of $50 \%$.

\section{RESULTS}

\section{Response Rate}

Ninety-three veterinarians (39\% response rate) from 51 clinics (63\% response rate) responded to the survey, with zero telephone responses. Information regarding the number of bovine clinics in the province was obtained through the College of Veterinarians of Ontario. One hundred sixty-five dairy producers $(27 \%)$ responded to the survey. Contact information was optional; $3 \%$ of veterinarians chose not to provide any contact information, whereas all of the producers surveyed gave at least one piece of contact information. No duplicate surveys were entered, and none of the respondents who began the first question of the survey failed to finish the survey.

\section{Descriptive Statistics}

Demographics. Veterinarian respondents had a mean age of $44(\mathrm{SE}=1.4)$, and $90 \%$ had attained their doctor of veterinary medicine from the Ontario Veterinary College (Guelph, Ontario, Canada); $70 \%$ of respondents were female. Respondents reported a mean of $51 \mathrm{~h}$ in the past year spent on continuing education $(\mathrm{SE}=7.6)$, and estimated a mean of $65 \%$ of their current workload was working with dairy cattle $(\mathrm{SE}=$ 2.9).

Producer respondents had a mean age of 43 ( $\mathrm{SE}=$ 1.0 ), with $66 \%$ having attended college or university; $81 \%$ of respondents were male. Forty-six percent had a freestall or pack barn, whereas $54 \%$ milked in a tiestall operation. Producers had a mean of 81 milking cows (range $=20-450, \mathrm{SE}=5.6$ ) at the time of the survey. Ninety percent of producers reported having a regular herd health visit with their veterinarian; $42 \%$ had these visits at least every 2 wk. All responding producers could be traced to 1 of the 15 DFO field (geographic) zones. The proportion of survey respondents from each zone did not differ significantly from the proportion of total dairy producers residing in that zone using a 2-way test of proportions.

Role of Veterinary Technicians. Seventy percent of veterinary clinics reported having a veterinary technician or registered veterinary technician on staff. Of clinics employing veterinary technicians, $87 \%$ reported one or more technicians on staff were registered with the Ontario Association of Veterinary Technicians (Guelph, Ontario, Canada). Of clinics with a veterinary technician, $49 \%$ did not have any clinic disbudding or dehorning done by technician. Twenty-five percent of clinics reported over $80 \%$ of clinic disbudding or dehorning was done by a technician.

Dehorning Procedures. Results are summarized in Tables 1 and 2. Veterinarians were asked to estimate what percentage of their disbudding or dehorning fell into the 3 age categories. Veterinarians performed a mean of $53 \%( \pm 4 \%)$ of their disbudding and dehorning with an electric burner; $31 \%( \pm 4 \%)$ with a gas-powered burner; and $14 \%( \pm 2 \%)$ by surgical amputation (Table $1)$. Veterinarians performed a mean of $39 \%( \pm 3 \%)$ of their disbudding and dehorning on calves less than 4 wk of age; $39 \%( \pm 3 \%)$ at 4 to 8 wk of age; and $22 \%$ $( \pm 3 \%)$ at more than 8 wk of age (Table 2). Ninety-four percent of responding veterinary clinics reported veterinarians or veterinary technicians performed disbudding and dehorning for a mean of $29 \%$ of their dairy clients. Ninety-eight percent reported training producers in performing disbudding and dehorning practices, including use of pain control.

Seventy-three percent of producers reported that either they or a member of their staff performed their disbudding or dehorning, with the remaining $27 \%$ reporting they had a veterinarian or technician perform this procedure. No producer respondents reported that they left horns intact on cattle on their farm. Producers performed a mean of $28 \%( \pm 4 \%)$ of their disbudding or dehorning on calves less than 4 wk of age; $36 \%( \pm 4 \%)$ 
Table 1. Percentage of calves (SE) disbudded or dehorned by different methods by veterinarians and producers in the Ontario (Canada) dairy industry

\begin{tabular}{lcc}
\hline Disbudding or dehorning method & $\begin{array}{c}\text { Mean percentage of } \\
\text { veterinarians using this method } \\
(\mathrm{n}=93)\end{array}$ & $\begin{array}{c}\text { Mean percentage of dairy } \\
\text { producers using this method } \\
(\mathrm{n}=118)\end{array}$ \\
\hline Large-diameter burner & $53(4)$ & $59(4)$ \\
Small-diameter burner & $31(4)$ & $18(3)$ \\
Surgical amputation & $14(2)$ & $13(3)$ \\
Caustic paste & 0 & $10(3)$ \\
Wire, saw, or keystone & $3(2)$ & 0 \\
\hline
\end{tabular}

at 4 to $8 \mathrm{wk}$ of age; and $36 \%( \pm 4 \%)$ at more than $8 \mathrm{wk}$ of age (Table 2).

The most commonly used method by producers was cautery with an electric, Rhinehart-style burner (59\%); followed by cautery with a small diameter, gas-powered burner (18\%); caustic paste (13\%); and surgical amputation by gouging (10\%; Table 1). An "other" method category was offered, but no respondents selected this category.

Producers using the large-diameter electric burner disbudded $22 \%$ of their calves at less than 4 wk of age, $38 \%$ at 4 to 8 wk of age, and $40 \%$ over 8 wk of age. Producers using a small-diameter burner disbudded $32 \%$ of their calves at less than $4 \mathrm{wk}$ of age, $59 \%$ at 4 to 8 wk of age, and $9 \%$ at over 8 wk of age. Caustic paste users disbudded $76 \%$ of their calves at less than 4 wk of age, $8 \%$ between 4 and 8 wk, and $16 \%$ over 8 wk of age.

Pain Control Practices. Results are summarized in Table 3. Veterinarians were asked a dichotomous outcome question on use of pain-control medication for each method and age group upon which they performed disbudding or dehorning. Ninety-seven percent of veterinarians reported using local anesthetic in 100\% of the calves they disbudded or dehorned.

A user of sedation or NSAID was defined as using it in $>50 \%$ of the animals disbudded or dehorned. The vast majority of veterinarians either use these medications for almost all or almost none of the animals they disbud or dehorn (Figure 1). Sixty-two percent of veterinarians used sedation; of these, $88 \%$ reported use $>90 \%$ of the time. Forty-eight percent of veterinarians used NSAID; of these, $92 \%$ reported use $>90 \%$ of the time. Of veterinarians using an NSAID, $89 \%$ cited their most commonly used NSAID as injectable meloxicam, whereas $7 \%$ used injectable flunixin meglumine and $4 \%$ chose injectable ketoprofen.

Sixty-two percent of producers who performed disbudding or dehorning themselves reported using local anesthetic (Table 3); $38 \%$ of producers reported using a sedative and $24 \%$ of producers reported using an NSAID. Of producers using an NSAID, 56\% used meloxicam injection, $26 \%$ used ketoprofen injection, and $18 \%$ chose flunixin meglumine injection.

Reasons for Medication Use and Lack of Use. Veterinarians' most commonly selected reasons for use of local anesthetic included ease of handling $(92 \%)$, concern for the welfare of the calf $(87 \%)$, lack of a cost barrier (86\%), and improved safety for both calf and handler (77\%). The most commonly selected reasons for use of NSAID (by NSAID users) included concern for the welfare of the calf $(100 \%)$, availability of an approved product (85\%), and low cost (49\%). The most commonly selected reasons by veterinarians not to use an NSAID (by NSAID nonusers) included concern over price $(92 \%)$, owner requests to not use NSAID other than price (50\%), and that pain control through NSAID use was not necessary for their disbudding or dehorning procedure $(18 \%)$. Of veterinarians using sedation, all (100\%) cited ease of handling as a reason for use, as well as safety (88\%), and lack of a cost barrier $(78 \%)$. Of those not using sedation, lack of need for handling (66\%) and owner request (38\%) were the most commonly cited reasons.

Among producers using local anesthetic, the most commonly selected reasons for use included concern for the welfare of the calf $(92 \%)$, ease of handling (81\%),

Table 2. Percentage of calves (SE) disbudded or dehorned at different ages by veterinarians and producers in the Ontario (Canada) dairy industry

\begin{tabular}{lcc}
\hline Age at disbudding or dehorning & $\begin{array}{c}\text { Mean percentage of calves disbudded } \\
\text { or dehorned by veterinarians } \\
(\mathrm{n}=93)\end{array}$ & $\begin{array}{c}\text { Mean percentage of calves disbudded } \\
\text { or dehorned by dairy producers } \\
(\mathrm{n}=118)\end{array}$ \\
\hline$<4 \mathrm{wk}$ & $39(3)$ & $28(4)$ \\
$4-8 \mathrm{wk}$ & $39(3)$ & $36(4)$ \\
$>8 \mathrm{wk}$ & $22(3)$ & $36(4)$ \\
\hline
\end{tabular}


Table 3. Percentage (SE) of Ontario (Canada)-based veterinarian and producer respondents reporting use of medications for the disbudding and dehorning of dairy calves

\begin{tabular}{lcc}
\hline Item & $\begin{array}{c}\text { Veterinarian users } \\
(\mathrm{n}=93)\end{array}$ & $\begin{array}{c}\text { Dairy producer users } \\
(\mathrm{n}=118)\end{array}$ \\
\hline Local anesthetic & $97(2)$ & $62(5)$ \\
Sedation & $62(5)$ & $38(5)$ \\
Nonsteroidal anti-inflammatory drug (NSAID) & $48(5)$ & $24(4)$ \\
\hline
\end{tabular}

and safety for both calf and handler (60\%). Although less commonly selected, some producers cited consumer concerns or marketplace demands as a reason they chose to use a local anesthetic (26\%) as well as information from the Code of Practice for the Care and Handling of Dairy Cattle (26\%). Of producers who did not use local anesthetic, the most commonly cited reason for lack of use was being unaware of this pain control option (48\%). Few producers cited concern over cost $(7 \%)$ or time $(14 \%)$ as being deterrents to using local anesthetic.

Of producers giving an NSAID for disbudding or dehorning, the most commonly cited reasons were concern for the welfare of the calf $(82 \%)$ and ease of availability $(50 \%)$. Twenty-eight percent of NSAID users reported consumer concerns or marketplace demands as a factor influencing their decision to use analgesia. Producers not choosing to use an NSAID for disbudding or dehorning most commonly reported they were unaware of this form of analgesia (45\%). Few producers cited concerns over cost (10\%), that this form of pain control

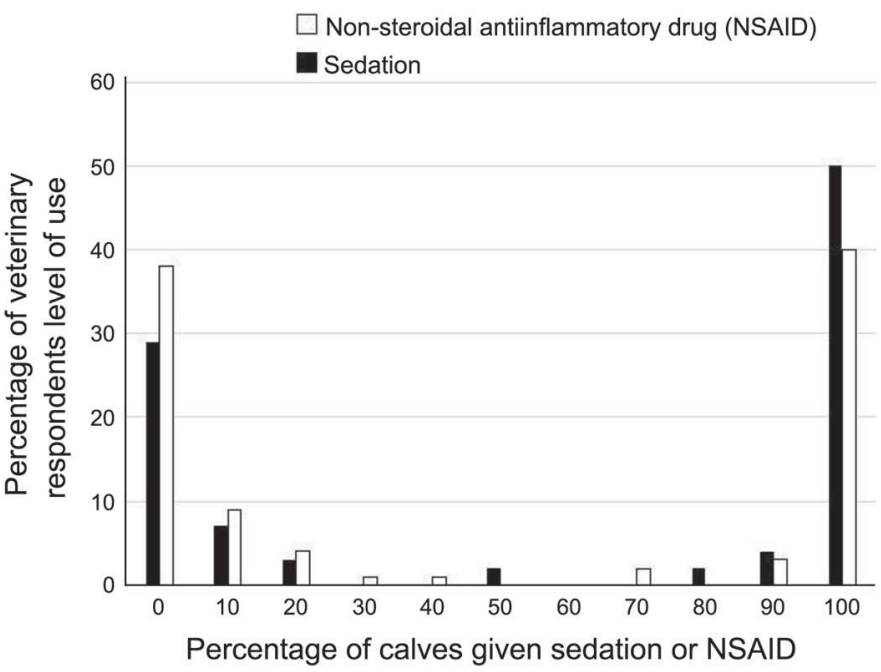

Figure 1. The appearance of a bimodal distribution using sedation and nonsteroidal anti-inflammatory drugs (NSAID) by veterinarians. Proportion of calves disbudded or dehorned by veterinarians given sedation or NSAID on the x-axis, with proportion of veterinarians selecting that value on the y-axis. Black bars represent sedation (xylazine) use, and white bars represent NSAID use. was not necessary (10\%), or that pain control in general was unnecessary $(10 \%)$.

Use of sedation by producers was commonly cited as being used to reduce handling stress; both ease of handling (86\%) and safety concerns $(75 \%)$ were cited. Producers also commonly believed use of sedation was important to control pain (86\%). Among producers not using sedation, few gave cited reasons, although a lack of need for handling was most commonly cited (21\%). A small number of producers identified sedation as not having a direct effect on disbudding or dehorning pain $(11 \%)$.

Reported Changes Over Time. Seventy-two percent of veterinarians practicing for more than $10 \mathrm{yr}$ reported changing disbudding and dehorning practices since 2004 . Of these veterinarians, $73 \%$ reported adding use of an NSAID; $32 \%$ reported adding use of local anesthetic; and $32 \%$ reported adding use of sedation.

Sixty-three percent of producers reported they had changed their disbudding or dehorning practices since 2004. Of these producers, $60 \%$ reported adding local anesthetic to their pain control protocol; $23 \%$ reported adding sedation; $17 \%$ reported adding an NSAID; and $30 \%$ reported performing these procedures at a younger age.

Influences on Change. Veterinarians who reported changing disbudding and dehorning practices since 2004 were asked an open-ended question as to why they changed their practices. The most commonly cited reason was concern for welfare of the calf $(75 \%)$. A smaller percentage included ease of handling as a reason for change $(22 \%)$.

Producers who reported changing their disbudding and dehorning practices since 2004 were asked who or what may have influenced their decision to change, with the option to select multiple responses or fill in an 'other' category. Seventy-three percent of producers selected their herd veterinarian as influential. The next 2 most commonly selected answers were knowledge from the Code of Practice for the Care and Handling of Dairy Cattle (22\%) and print publications (18\%).

Perceptions of Pain: Likert Scoring. Results are summarized in Table 4. We found were no significant differences between producers and veterinarians for the same procedure or condition in equality of distribution, 
Table 5. Multivariate logistic regression model of factors associated with use of local anesthetic use by dairy producers for disbudding or dehorning

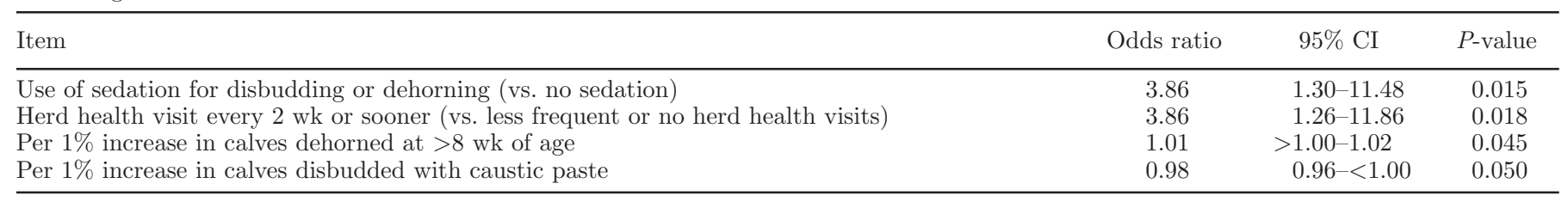

with 7 times greater odds of using sedation. Producers who reported using the social media platform Twitter (www.twitter.com) for business purposes had 4.4 times greater odds of using sedatives for dehorning as compared with those who did not use this platform $(P$ $=0.03)$. Increased perception of dystocia pain was posi- tively associated with use of sedation for disbudding or dehorning (odds ratio $=1.4 ; P=0.04$ ). A producer who scored dystocia 2 Likert units higher would be twice as likely to use sedatives. Producers who had a herd health visit by their veterinarian at least every 2 wk tended to have increased odds of using sedatives (odds

$\square$ Producer respondents

- Veterinarian respondents
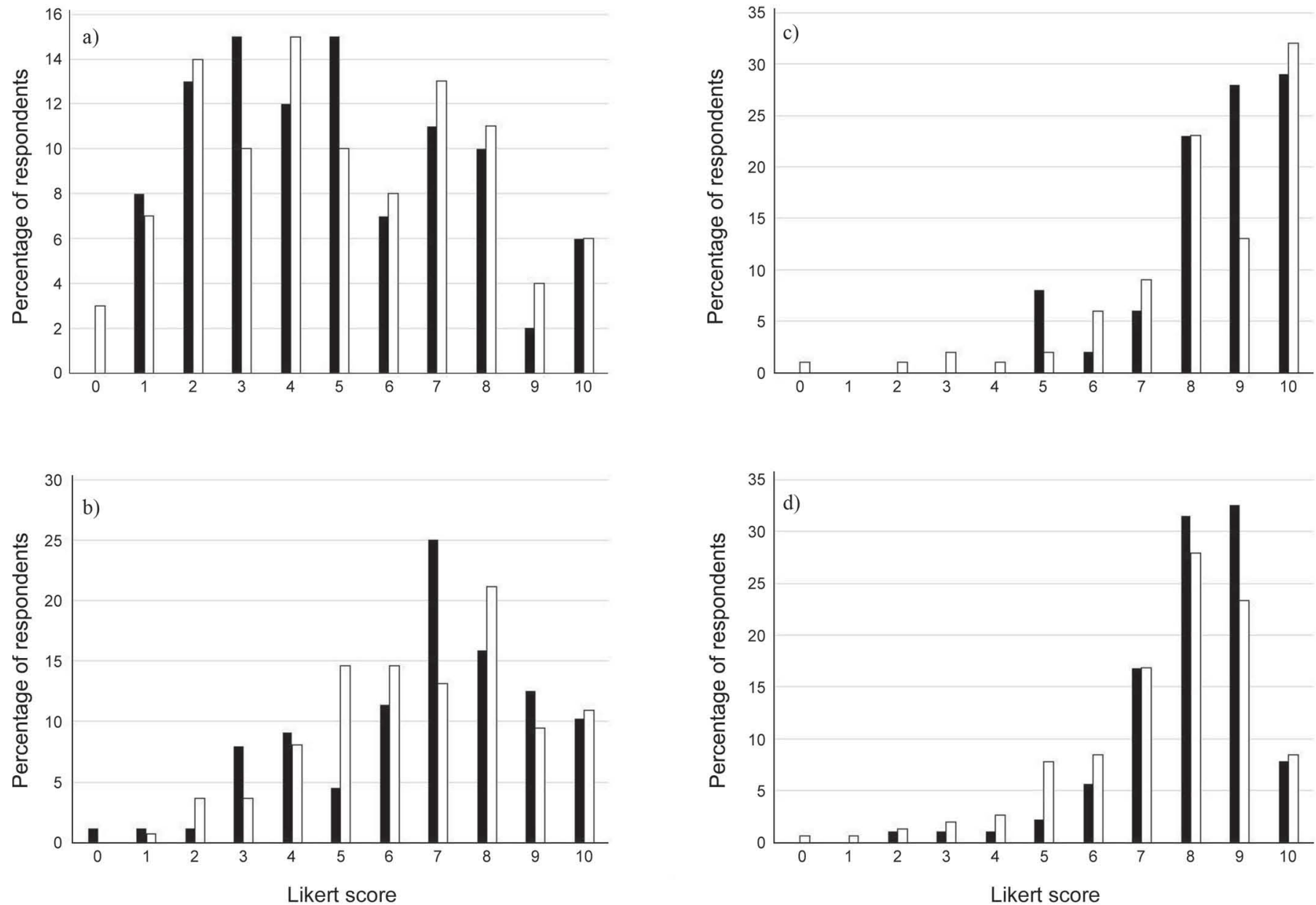

Figure 2. Distribution of Likert scores for the perceived painfulness of different procedures or conditions by veterinarians (black bars) and dairy producers (white bars). All procedures or conditions were defined as having been completed without the use of a pain control. Likert scores $(0=$ not causing pain, $10=$ worst pain imaginable) are on the $\mathrm{x}$-axes, and the percentage of respondents choosing that score are on the $\mathrm{y}$-axes. (A) Disbudding at $2 \mathrm{~d}$ of age with caustic paste; (B) disbudding at 2 wk of age by small diameter disbudding iron; (C) dehorning at 5 mo of age by gouging and cautery; (D) dystocia, defined as a hard pull with a calving jack. 
Table 6. Multivariate logistic regression model of factors associated with use of sedation by dairy producers for disbudding or dehorning

\begin{tabular}{|c|c|c|c|}
\hline Item & Odds ratio & $95 \%$ CI & $P$ value \\
\hline Twitter use (professional) & 4.39 & $1.16-16.59$ & 0.029 \\
\hline Producer perception of the pain of dystocia (per one point increase) & 1.43 & $1.02-2.01$ & 0.036 \\
\hline
\end{tabular}

ratio $=2.5 ; P=0.071)$. At a probability threshold of 0.5 , the local model correctly classified $73 \%$ of the data, with a specificity of $79 \%$ and a sensitivity of $64 \%$. Area under the ROC curve was considered good, at 0.81 .

\section{DISCUSSION}

\section{Respondents and Nonresponse Bias}

Producer respondents were slightly younger, with a mean age of 43, than the largest segment of dairy producer population, which is between 45 to 54 yr of age (Canadian Dairy Commission, 2016). Farm size, with a mean of 81 cows, was comparable to the national average of 77 cows (Canadian Dairy Commission, 2016).

Although risk of response bias is present in all surveys, especially those involving sensitive topics such as pain control use on farms, a lack of anonymous respondents or dropouts in the producer survey may suggest that this effect was small within our sample. If reluctance to report lack of pain control use for disbudding and dehorning was a concern, we would expect to see a proportion of producers decline to provide contact information, or decline to finish the survey once questions on pain control were posed. However, all producers provided at least one piece of contact information, and all respondents who answered the first question completed the survey. Answers were similar between producers and veterinarians when examining the proportion using a veterinarian or technician for these procedures (27\% of dairy producers reported using a veterinarian; veterinarians estimated performing dehorning for $29 \%$ of their clients). We would expect to see over-representation of veterinarian or technician dehorned farms in the producer survey (as those farms are more likely to use pain control) if those not using pain control disproportionately chose not to respond to the survey. However, it is still likely that the reported results do not entirely capture the adoption of pain control use in the source population.

\section{Use of Veterinarians or Technicians by Producers for Dehorning}

In our survey populations, the proportion of disbudding or dehorning performed by veterinarian or technician was similar to findings in a 2004 survey by Misch et al. (2007), where veterinarians estimated they performed this task for $31 \%$ of their clients, and $22 \%$ of dairy producers reported using a veterinarian. Although training a staff member to perform this task may potentially be the most cost-effective option, labor is a limiting resource on many farms (Findeis, 2002), and paying a professional already skilled in this area may be easier for some producers. Another potentially more cost-effective option is for veterinary clinics is to employ technicians to perform this task, which can be coupled to other value-added services on-farm. Almost half of responding veterinary clinics did not use technicians for any disbudding or dehorning, but $26 \%$ reported technicians performing $>80 \%$ of all the disbudding and dehorning that the clinic performed. Whereas data on dehorning by technicians have not previously been explored, based on the experiences of our research group, we feel the number of Ontario clinics utilizing technicians for these procedures has increased over time.

\section{Veterinary Survey}

It was encouraging to see that $78 \%$ of veterinary disbudding or dehorning occurs before 8 wk of age, which indicates these calves would have been disbudded rather than dehorned, and would experience less pain than animals dehorned by amputation at older ages (Petrie et al., 1996). Our results suggest the use of pain control by veterinarians has changed over the past decade. The use of local anesthetic in our study was $97 \%$, as compared with $77 \%$ found previously by Misch et al. (2007). Use of NSAID by veterinarians appeared to increase dramatically; $48 \%$ of veterinarians used this medication in our study as compared with $2 \%$ reporting use previously by Misch et al. (2007). Use of an NSAID is beneficial for disbudding and dehorning procedures; when local anesthesia alone is used the initial cortisol rise and behavioral indicators of pain are delayed for the duration of the block, but a delayed response occurs without NSAID administration (Sylvester et al., 1998; Grøndahl-Nielsen et al., 1999; Stewart et al., 2009; Heinrich et al., 2010).

Interestingly, both NSAID and sedative use by individual veterinarians appeared dichotomous; that is, a practitioner either did not use the medication at all 
or used it in the vast majority of their disbudding or dehorning (Figure 1). This is promising for future adoption of NSAID use: if a practitioner can be convinced to use an analgesic, it is likely they will then use it in the majority of their disbudding and dehorning.

Cited reasons for pain control use by veterinarians were primarily animal-centered, namely concern for the welfare and handling of the calf undergoing the procedure, similar to recent findings from Ventura et al. (2015). Recommendations for use by governing bodies or concern for perceptions of the industry were not commonly selected. This may be a result of veterinarians in our survey population primarily viewing pain control for disbudding and dehorning as an individual animal issue. If veterinarians believe the procedure causes pain that can be mitigated by either local anesthetics or NSAID use, they appear to use these products on the majority of animals they disbud or dehorn.

Whereas the differences seen are postulated to have occurred due to veterinarians changing their disbudding or dehorning practices, it is also possible that different pools of veterinarians were surveyed now, as compared with those responding to the 2004 survey by Misch et al. (2007). We attempted to answer this question by asking veterinarians that had practiced for $10 \mathrm{yr}$ or more if their practices had changed over this time period. Because nearly three-quarters of respondents to this question reported changes, the differences between survey years likely reflect individual veterinarians changing practices as opposed to new veterinarians with improved pain-control practices replacing others in the population. Similar to the reasons cited for paincontrol drug use, the most commonly selected reason for having made a change in disbudding or dehorning practices was concern for the welfare of the individual calf. Whereas older studies have found younger (Raekallio et al., 2003) and female (Huxley and Whay, 2006) veterinarians are more sensitive to pain in animals, our results showing a large proportion of veterinarians in practice over $10 \mathrm{yr}$ changing their practices suggests that perhaps extension efforts have had an effect, or perhaps the practices of younger veterinarians have influenced their older colleagues.

\section{Producer Survey}

Our results suggest producers are disbudding calves at a younger age as compared with results from Misch et al. (2007). Producers were disbudding more calves with a small-diameter, gas-powered burner (18\% in this study compared with $4 \%$ in Misch et al., 2007), and correspondingly fewer with surgical amputation methods (gouging, saw, or keystone dehorning shears; $13 \%$ in this study compared with $32 \%$ in Misch et al.,
2007). This is consistent with the $63 \%$ of producers in our survey who reported changed their disbudding and dehorning practices in the past $10 \mathrm{yr}, 30 \%$ of whom reported performing these procedures at a younger age. Despite these advances, more than one-third of our respondents reported disbudding or dehorning at more than 8 wk of age, similar to what was found in 2004 (Misch et al., 2007). Although increased use of less invasive methods suggest this third of producers is performing dehorning at a younger age (e.g., $12 \mathrm{wk}$ instead of $6 \mathrm{mo}$ ), it is still beyond the Canadian recommendations and will involve more tissue damage than disbudding at a younger age. Although disbudding at less than $3 \mathrm{wk}$ is recommended (National Farm Animal Care Council, 2009), these tasks are likely not the most enjoyable, and may be pushed back to a later date when labor is limited or when more pressing jobs are at hand.

An increase in the use of local anesthetic, sedatives, and NSAID for disbudding and dehorning was seen, all of which are higher than previously reported in North America-based surveys (18\%, Hoe and Ruegg, 2006; $22 \%$, Misch et al., 2007; 12\%, Fulwider et al., 2008; $45 \%$, Vasseur et al., 2010). The dramatic rise in the adoption of NSAID among producers has not been previously reported. Concern for the individual animal was a common theme when users of these medications were asked why they had chosen them. Although industrycentered reasons were less commonly reported, it is interesting that over one-quarter of producers cited consumer concerns or marketplace demands as important reasons for choosing to use both local anesthetics and NSAID. Increased awareness of these concerns by dairy producers can only help bridge gaps between consumer expectations and on-farm practices.

We noted an association of age and method of disbudding or dehorning with pain control use: producers who disbudded or dehorned animals at $>8$ wk of age were more likely to use local anesthetic. Use of local anesthetic in older cattle may be a reflection of the difficulty handling larger animals; $81 \%$ of local anesthetic users selected ease of handling as important reason in their decision to use this medication. It may also be a result of older animals potentially displaying more behavioral indicators of pain; although no studies have directly compared these indicators among calves of different ages, it may be easier to recognize pain in older calves. For example, inert lying behavior seen in 2 caustic paste trials, which confounded typical behavioral indicators of pain, was postulated to be either a result of the method used or due to the age of the calf (Stilwell et al., 2007, 2009). The lack of use of local anesthetic for caustic paste disbudding reported here may reflect that the pain caused by caustic paste is suggested not to be as immediate as cautery, as there 
is less struggling during the procedure (Stilwell et al., 2009). Producers using this method and leaving within minutes of application may not witness the pain-related behaviors that calves disbudded with this method exhibit when performed without anesthesia or analgesia (Stilwell et al., 2007, 2009).

Use of xylazine sedation by producers was significantly associated with increased perception of the pain of dystocia. It is possible that more empathetic producers prefer calves to experience less handling stress during disbudding or dehorning. Producers who are more sensitive to pain take the pain of dehorning more seriously (Wikman et al., 2013). However, we observed an association of perceived pain of dystocia with use of sedatives, but not with use of local anesthetic. Perception of pain for the 3 disbudding and dehorning procedures was not related to local anesthetic or sedation use.

The association between Twitter use (a social media platform) for professional purposes was also associated with use of sedation for disbudding, but age and education level were not. It may be that typical indicators of greater awareness of currently recommended management procedures (e.g., producers of a younger age, or with a higher level of education) are no longer appropriate measures in 2016. Based on our results, producers who are more connected to information sources, or perhaps like-minded peers (i.e., those who report using social media as a business tool), are perhaps more likely to be aware of different options or management strategies, and that this connection to information is independent of age and education level.

\section{Perceptions of Pain}

It was remarkable how similarly veterinary and producer survey respondents viewed pain in cattle. The 2 survey populations did not differ in the rating of any of the 8 conditions or procedure in both the comparison of means and ranges. The 2 practices or conditions deemed to be most painful (dystocia and gouging and burning at $5 \mathrm{mo}$ ) also contained the least apparent variation in distribution. Dehorning at an older age was similarly found to be one of the most painful procedure in a survey of US veterinarians (Fajt et al., 2011), but, unlike our survey, was ranked lower than a leftdisplaced abomasum in a survey of UK veterinarians (Huxley and Whay, 2006). That said, it is difficult to compare perceptions of pain between studies, not only because the populations and methods differ, but the list of procedures and conditions given to participants differed in our work and may confound comparisons between studies.
Familiarity of both producers and veterinarians to both surgical amputation dehorning and dystocia may explain the tighter distribution within our survey, as compared with the other procedures or conditions listed. Although cautery (alone, without gouging) is the most commonly performed disbudding practice, found both in our survey as well as others (Hoe and Ruegg, 2006; Fulwider et al., 2008), it is likely producers and veterinarians are familiar with the procedure of gouging, with producers perhaps having historically used this method. Dystocia is also clearly a condition that the vast majority of producers and veterinarians (if not all) are familiar with. In contrast, disbudding by caustic paste is much less commonly used, and some debate exists as to the nature and duration of the pain involved, as well as far fewer clinical trials examining pain control (Vickers et al., 2005; Stilwell et al., 2007, 2009). As this procedure had the widest apparent range of variation in both populations, we postulated that this reflects the lack of definitive conclusions with respect to the nature and duration of pain caused by caustic paste, as well as a lack of familiarity of respondents with this practice.

\section{Veterinary Involvement and Use of Pain Control}

Veterinary influence on producers' use of pain control for disbudding and dehorning was seen both in the results directly in the producer survey, as well as in the influence of herd health frequency in the predictive models. Essentially all veterinarians surveyed in the present study reported use of a local anesthetic; however, this was not the case $10 \mathrm{yr}$ ago (Misch et al., 2007). Perhaps the increased adoption by veterinarians over this period is one of the major factors associated with the increased producer use. Interestingly, current NSAID use by veterinarians (62\%) and producers $(24 \%)$ is not unlike that of local anesthetic use reported by Misch et al. (2007) by both veterinarians (77\%) and producers $(21 \%)$; if NSAID use by veterinarians rose to full adoption, we wonder if a similar upswing in producer use of NSAID may be seen. It would be difficult to increase use by producers if the medication is seen as unimportant (and therefore unused) by their veterinarian.

In this vein, it is interesting that producer concerns regarding cost of NSAID were not commonly cited as a roadblock to analgesic use; rather, lack of awareness was. Although it may be more palatable to plead ignorance over frugality when asked why not to use analgesia for a painful procedure, only $59 \%$ of veterinarians used NSAID, and the vast majority of these started using these products within the past $10 \mathrm{yr}$. All NSAID products in Canada require veterinary prescription. As 
one may argue that producers rely on their veterinarian for information regarding pain management for disbudding or dehorning, previous lack of veterinary use of NSAID may have precluded producer adoption of these products. The mismatch between veterinarians' concerns over NSAID cost (92\% of veterinary NSAID nonusers) and producer concerns over NSAID cost (reported by $10 \%$ of producer NSAID nonusers) merits further consideration. Although it is possible producers self-censored their economic concerns, it is also possible that veterinarians pre-emptively do not offer or discuss NSAID use if they, perhaps wrongly, anticipated producers' economic concerns. Concern by veterinarians regarding cost, and the influence of this on pain management use, is similar to results found in a national study by Hewson et al. (2007). Unlike local anesthesia, the effects of NSAID administration do not provide any handling benefit. Although concern for pain control was commonly cited in our survey as a reason to use local anesthetic (87\%), ease of handling was also seen as important (92\%). Previous work found tractability was often seen as a reason for local anesthetic use by food animal veterinarians, as opposed to concern for pain control (Morgan, 2009).

Producers who had changed disbudding or dehorning practices in the past $10 \mathrm{yr}$ highlighted the importance of their herd veterinarian in such changes. The influence of veterinary involvement was also seen regarding use of pain control. Producers with a herd health visit at least biweekly were nearly 4 times more likely to use local anesthetic. Although the frequency of herd visits is generally associated with herd size, we did not find associations of herd size with veterinary visit frequency or use of pain-control.

\section{Limitations and Considerations}

Though both our study and that of Misch et al. (2007) used similar source populations for both dairy producers and veterinarians, this was not a longitudinal study and differences between study years may only be compared numerically. Differences in response bias may exist between the 2 survey years.

Producers were asked about medication use in the majority of calves; variation of use on a single farm could result in a lower proportion of total calves receiving pain control. However, we assumed practices were likely to be similar between calves within a farm based on our experience with this population.

Factors that appear to have influenced veterinarians and dairy producers to adopt best practices may not necessarily be indicative of what may motivate change among current nonadopters. These populations (adopters and nonadopters) may have other differences not elicited in this work, which may factor into their willingness to change disbudding and dehorning practices.

\section{CONCLUSIONS}

This work demonstrates improvements in use of pain control for disbudding and dehorning by both veterinarians and dairy producers in Ontario, Canada, from previous published work in North America. The most commonly reported reason for the use of anesthesia and analgesia was concern for the welfare of the individual calf, although it is interesting to note that a quarter of producers also identified consumer attitudes as directly affecting their decision to use pain control. Producers and veterinarians viewed the experience of pain by cattle similarly, which should facilitate increased adoption of best management practices for these painful procedures. Veterinary involvement was highly influential on adoption of pain-control practices by producers. However, a disconnect in identifying roadblocks to increased pain control adoption rates exits between veterinarians and producers, as seen by the discrepancy between the 2 groups regarding cost being prohibitive for NSAID use. A fuller examination of motivating factors to request, dispense, and discuss information on best practices by both parties should be undertaken to better determine how to further improve pain control adoption for the disbudding and dehorning of dairy calves. We encourage veterinarians to advocate fuller implementation of pain control practices by producers for disbudding calves.

\section{ACKNOWLEDGMENTS}

This project was funded by a Knowledge Translation and Transfer grant from the Agri-Food and Rural Link program of the Ontario Ministry of Agriculture, Food, and Rural Affairs-University of Guelph partnership (Guelph, Ontario, Canada).

\section{REFERENCES}

American Veterinary Medical Association (AVMA). 2014. Literature review on the welfare implications of the dehorning and disbudding of cattle. Accessed Apr. 1, 2016. https://www.avma.org/KB/ Resources/LiteratureReviews/Pages/Welfare-Implications-ofDehorning-and-Disbudding-Cattle.aspx.

Braz, M., M. Carreira, N. Carolino, T. Rodrigues, and G. Stilwell. 2012. Effect of rectal or intravenous tramadol on the incidence of pain-related behavior after disbudding calves with caustic paste. Appl. Anim. Behav. Sci. 136:20-25.

Canadian Dairy Commission. 2016. The Industry: Production. Accessed Apr. 1, 2016. http://www.cdc-ccl.gc.ca/CDC/index-eng. php?id $=3801$.

Canadian Veterinary Medical Association. 2010. Disbudding and dehorning of cattle-Position statement. Accessed Apr. 1, 2016. http://www.canadianveterinarians.net/documents/disbuddingand-dehorning-of-cattle. 
Dairy Farmers of Canada. 2015. proAction: Leading the way for sustainable dairy farming; providing assurance to customers about farm practices. Dairy Farmers of Canada, Ottawa, Ontario, Canada. Accessed Apr. 1, 2016. https://www.dairyfarmers.ca/Media/ Files/proaction/proaction_ang_lr15.pdf

Duffield, T. F., A. Heinrich, S. T. Millman, A. DeHaan, S. James, and K. Lissemore. 2010. Reduction in pain response by combined use of local lidocaine anesthesia and systemic ketoprofen in dairy calves dehorned by heat cauterization. Can. Vet. J. 51:283-288.

Fajt, V. R., S. A. Wagner, and B. Norby. 2011. Analgesic drug administration and attitudes about analgesia in cattle among bovine practitioners in the United States. J. Am. Vet. Med. Assoc. 238:755-767.

Findeis, J. L. 2002. Hired farm labor adjustments and constraints. Pages 3-14 in The Dynamics of Hired Farm labor: Constraints and Community Responses. J. L. Findeis, A. M. Vandeman, J. M. Larson, J. L. Runyan, ed. CABI Publishing, New York, NY.

Fulwider, W. K., T. Grandin, B. E. Rollin, T. E. Engle, N. L. Dalsted, and W. D. Lamm. 2008. Survey of dairy management practice on one hundred thirteen north central and northeastern United States dairies. J. Dairy Sci. 91:1686-1692.

Gottardo, F., E. Nalon, B. Contiero, S. Normando, P. Dalvit, and G. Cozzi. 2011. The dehorning of dairy calves: Practices and opinions of 639 farmers. J. Dairy Sci. 94:5724-5734.

Grøndahl-Nielsen, C., H. B. Simonsen, J. Damkjer Lund, and M. Hesselholt. 1999. Behavioural, endocrine and cardiac responses in young calves undergoing dehorning without and with use of sedation and analgesia. Vet. J. 158:14-20.

Heinrich, A., T. F. Duffield, K. D. Lissemore, and S. T. Millman. 2010. The effect of meloxicam on behavior and pain sensitivity of dairy calves following cautery dehorning with a local anesthetic. J. Dairy Sci. 93:2450-2457.

Hewson, C. J., I. R. Dohoo, K. A. Lemke, and H. W. Barkema. 2007. Factors affecting Canadian veterinarians' use of analgesics when dehorning beef and dairy calves. Can. Vet. J. 48:1129-1136.

Hoe, F. G., and P. L. Ruegg. 2006. Opinions and practices of Wisconsin dairy producers about biosecurity and animal well-being. J. Dairy Sci. 89:2297-2308.

Hokkanen, A. H., I. Wilkman, T. Korhonen, M. Pastell, A. Valros, O. Vainio, and L. Hänninen. 2015. Perceptions and practices of Finnish dairy producers on disbudding pain in calves. J. Dairy Sci. 98:823-831.

Huxley, J. N., and H. R. Whay. 2006. Current attitudes of cattle practitioners to pain and the use of analgesics in cattle. Vet. Rec. 159:662-668

Misch, L. J., T. F. Duffield, S. T. Millman, and K. D. Lissemore. 2007. An investigation into the practices of dairy producers and veterinarians in dehorning dairy calves in Ontario. Can. Vet. J. 48:1249-1254.

Morgan, C. A. 2009. Stepping up to the plate: Animal welfare, veterinarians, and ethical conflicts (Doctoral dissertation). Accessed Apr. 1, 2016. https://open.library.ubc.ca/cIRcle/ collections/ubctheses/24/items/1.0067398

National Farm Animal Care Council. 2009. Code of Practice for the Care and Handling of Dairy Cattle. Ottawa, Ontario, Canada.

Petrie, N. J., D. J. Mellor, K. J. Stafford, R. A. Bruce, and R. N. Ward. 1996. Cortisol responses of calves to two methods of disbudding used with or without local anaesthetic. N. Z. Vet. J. 44:9-14.
Raekallio, M., K. M. Heinonen, J. Kuussaari, and O. Vainio. 2003. Pain alleviation in animals: Attitudes and practices of Finnish veterinarians. Vet. J. 165:131-135.

Robbins, J. A., D. M. Weary, C. A. Schuppli, and M. A. G. von Keyserlingk. 2015. Stakeholder views on treating pain due to dehorning dairy calves. Anim. Welf. 24:399-406.

Spooner, J. M., C. A. Schuppli, and D. Fraser. 2014. Attitudes of Canadian citizens toward farm animal welfare: A qualitative study. Livest. Sci. 163:150-158.

Stafford, K. J., and D. J. Mellor. 2011. Addressing the pain associated with disbudding and dehorning in cattle. Appl. Anim. Behav. Sci. 135:226-231

Staněk, S., V. Zink, O. Dolezal, and L. Stolc. 2014. Survey of preweaning dairy calf-rearing practices in Czech dairy herds. J. Dairy Sci. 97:3973-3981.

Stewart, M., J. M. Stookey, K. J. Stafford, C. B. Tucker, A. R. Rogers, S. K. Dowling, G. A. Verkerk, A. L. Schaefer, and J. R. Webster. 2009. Effects of local anesthetic and a nonsteroidal antiinflammatory drug on pain responses of dairy calves to hot-iron dehorning. J. Dairy Sci. 92:1512-1519.

Stilwell, G., R. C. de Carvalho, M. S. Lima, and D. M. Broom. 2009. Effect of caustic paste disbudding, using local anaesthesia with and without analgesia, on behaviour and cortisol of calves. Appl. Anim. Behav. Sci. 116:35-44.

Stilwell, G., M. S. Lima, and D. M. Broom. 2007. Comparing the effects of three different disbudding methods on behaviour and plasma cortisol of calves. Rev. Port. Cienc. Vet. 102:281-288.

Stock, M. L., S. L. Baldridge, D. Griffin, and J. F. Coetzee. 2013. Bovine Dehorning: Assessing pain and providing analgesic management. Vet Clin. North Am. Food Anim. Pract. 29:103-133.

Sylvester, S. P., D. J. Mellor, K. J. Stafford, R. A. Bruce, and R. N. Ward. 1998. Acute cortisol responses of calves to scoop dehorning using local anaesthesia and/or cautery of the wound. Aust. Vet. J. $76: 118-122$

United States Department of Agriculture. 2009. Dairy 2007: Part $\mathrm{V}$ : Changes in dairy cattle health and management practices in the United States, 1996-2007. USDA-APHIS National Animal Health Monitoring System. Accessed Apr. 1, 2016. http://www. aphis.usda.gov/animal_health/nahms/dairy/downloads/dairy07/ Dairy07_dr_PartV_rev.pdf.

Vasseur, E., F. Borderas, R. I. Cue, D. Lefebvre, D. Pellerin, J. Rushen, K. M. Wade, and A. M. de Passille. 2010. A survey of dairy calf management practices in Canada that affect animal welfare. J. Dairy Sci. 93:1307-1315.

Ventura, B. A., M. A. G. von Keyserlingk, and D. M. Weary. 2015. Animal welfare concerns and values of stakeholders within the dairy industry. J. Agric. Environ. Ethics 28:109-126.

Vickers, K. J., L. Neil, L. M. Kiehlbauch, and D. M. Weary. 2005. Calf response to caustic paste and hot-iron dehorning using sedation with and without local anesthetic. J. Dairy Sci. 88:1454-1459.

Wikman, I., A. H. Hokkanen, M. Pastell, T. Kauppinen, A. Valros, and L. Hänninen. 2013. Dairy producer attitudes to pain in cattle in relation to disbudding calves. J. Dairy Sci. 96:6894-6903. 